\title{
THEORETICAL MODEL OF THE VISIBILITY LEVEL AND PRACTICAL MEANS OF ITS IMPLEMENTATION
}

\author{
M. ZALESIŃSKA \\ Poznan University of Technology, Institute of Electrical Engineering and Electronics, Poland.
}

\begin{abstract}
Driving a car, especially in city traffic, is a greatly complex process combining observation, recognition and psychomotoric functions. Safe, efficient and comfortable driving requires a specific level of visibility of road obstacles. The difficulty in spotting an obstacle in the road and in evaluating its effect on driving depends on such factors as lighting conditions in the road and its vicinity, presence of sources of glare, sources of distracting and attracting attention in the driver's field of vision, for example, electronic outdoor advertising boards (LED billboards), the obstacle's geometric and photometric properties, observation conditions and the driver's visual performance. The research on the visibility of obstacles in the road has shown that the satisfaction of normative requirements in relation to average luminance and the general and longitudinal uniformity does not guarantee that an obstacle will be spotted. Thus, it is necessary to introduce another criterion to make it possible to evaluate the visibility of obstacles in the road. Visibility formula was described by Adrian in 1989 and applied with visibility levels in North America as quality criterion. For the purposes of designing road lighting systems, the visibility criterion is not used in European countries yet. Due to simplifications, other standards and requirements, it is also impossible to directly employ the visibility criterion used in United States, namely the Small Target Visibility, based to a large extent on Adrian's visibility model.

Keywords: Road lighting, road lighting standards, Small Target Visibility, visibility level.
\end{abstract}

\section{INTRODUCTION}

Once dusk sets in, human visibility performance drops significantly. Details cannot be distinguished any more. Only the general outlines of objects become recognizable. Visibility sharpness and colour recognition ability worsen. At such times, humans use selected already stored and remembered information to improve performance. However, the same level of perception and efficiency of navigating the surroundings as during the day cannot be achieved that way. It is crucial to create the right viewing conditions in any situation that requires a specific visual task to be performed quickly, accurately, safely and at no excessive effort.

Illuminating outdoor areas gains special importance in case of municipal roads and areas, producing both social and economic benefits. Illuminated municipal areas increase the sense of safety among residents and tourists, partly due to limited crime and vandalism. It also promotes social activities and raises the city's attractiveness. Road lighting mainly impacts the quality of vision of the participants of main traffic lines, reduces the number and severity of accidents, increases traffic fluency and capacity of roads.

Research projects in many countries show that properly executed road lighting may contributed to the reduction of the number of accidents at night by about $20-40 \%$ in relation to situations where the road lighting is not present, is switched off or is poorly designed [1]. A great deal of investment and operating costs of road lighting equipment translate to savings to the society, as many accidents are effectively prevented.

\section{DRIVER'S VISUAL TASKS}

To efficiently drive a vehicle, especially in city traffic, its driver needs plenty of visual information about the surroundings. The driver must be able to distinguish the outline of the road, pedestrian crossings, sidewalks, road traffic signs and lights, orientation points, the presence of other traffic 


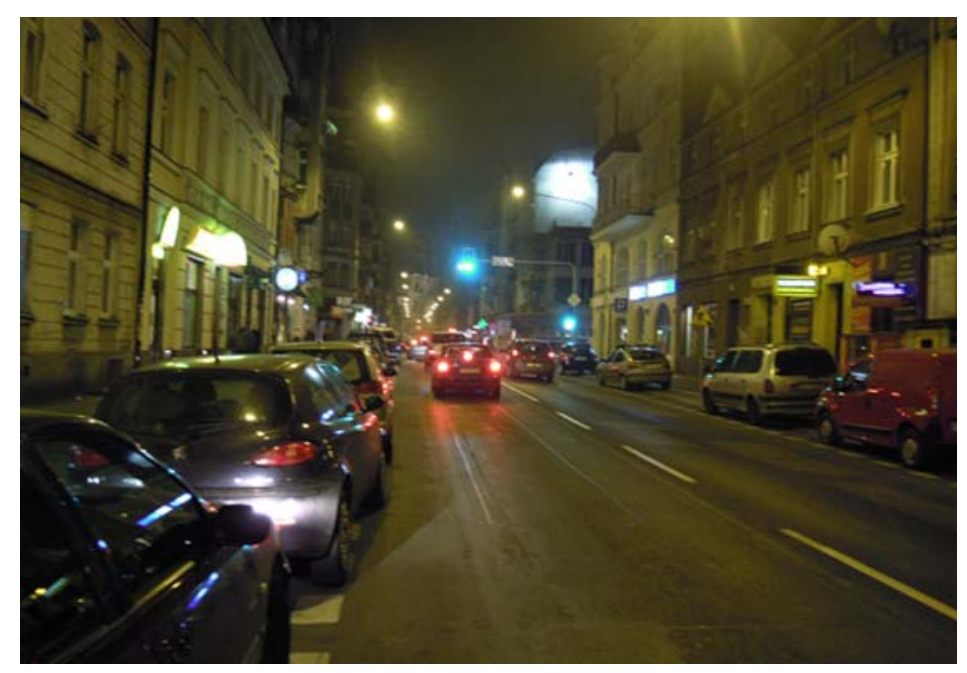

Figure 1: Example of a typical road situation in municipal traffic.

participants, their location, speed and direction of movement. Moreover, all information must be received in the right moment, so that the brain can identify and process it and subsequently make a correct decision. Figure 1 presents an example of a road situation in municipal traffic, where the driver is to perform a visual task and only has a very limited time to make a decision.

The spotting of an object related to the process of driving results in driver's activities consisting in either just its observation or its observation followed by making a manoeuvre. Hence, three levels of performing the visual task should be applied to its investigation: the position level, the situational level and the navigational level. The position level applies to maintaining the desired position and the correct course in a traffic lane. The situational level is connected to making manoeuvres in the road and maintaining and adjusting the vehicle's speed. The navigational level is related to orientation in the surroundings and selection of the route. Each of the above levels requires special information from the visual analysis of the road and its vicinity that allows correct execution of a specific task. To maintain the right position in a traffic lane, it is important to visually recognize changes in the roadway, such as traffic lane lines, curbs, shoulders, etc., and to spot the relative movement of the surroundings. During daytime, this part of visual information is received automatically thanks to peripheral vision and does not require increased attention from the driver. Once dusk sets in, especially if only the vehicle's headlights are used, the field of vision is greatly limited and so is the volume of visual information that reaches the driver. In such conditions, the driver's attention is generally focused only on the correct position in the lane and the driver's visual tasks are, out of necessity, limited to spotting changes in the road's vicinity. If, apart from the vehicle's headlights, the road's fixed lighting is operating, providing more information about its surroundings, additional, more complex driving-related activities at the situational and navigational level can be performed. Visual tasks at the situational level are related to assessing the current position and speed in relation to other traffic participants. It is necessary to have visual information indicating the need to adjust the speed, driving direction or position in the road. The driver must recognize typical features of a given situation, relying, to a large extent, on previously gathered experience and knowledge and, if necessary, must make the right manoeuvre. Visual tasks at the 
navigational level are connected with finding and processing information from the direct or nondirect vicinity of the road - simple and quick recognition of characteristic points in the surroundings, traffic signs, information boards, street names and parcel numbers, etc., required to make decisions on the choice of route. In this situation, the illumination of areas adjacent to the road is important, since the driver's vision is also directed outside the road. The completion of a visual task at the navigational level is only possible in favourable vision conditions at the position and situational levels.

\section{VISIBILITY OF TARGETS IN ILLUMINATED ROADS}

A driver can spot an object in the road or in its background only if the contrast the object creates with the background (the road or its background) is above the threshold value of the contrast. If the object's luminance is higher than the luminance of the background the contrast is positive, otherwise the contrast is negative - which is most often the case with road lighting (see Fig. 2).

The difficulty of spotting obstacles in the road depends on the following factors:

- The contrast between the luminance of the object and its immediate visual background,

- the general level of adaptation of that portion of the retina of the eye concerned with the object,

- observer duration on road,

- the size, shape of the object,

- disability glare - the amount of veiling luminance entering the eye,

- transient adaptation - the difference in eye adaptation between successive eye movements,

- the background complexity and the dynamics of traffic and

- visual capability of drivers.

Numerous research projects for the past 70 years aimed to identify the criterion for evaluation of visibility of obstacles in the road. On the basis of Blackwell's laboratory research, the International Commission on Illumination (CIE) introduced in 1972 the Visibility Level (VL) (eqn (1)) defined as the

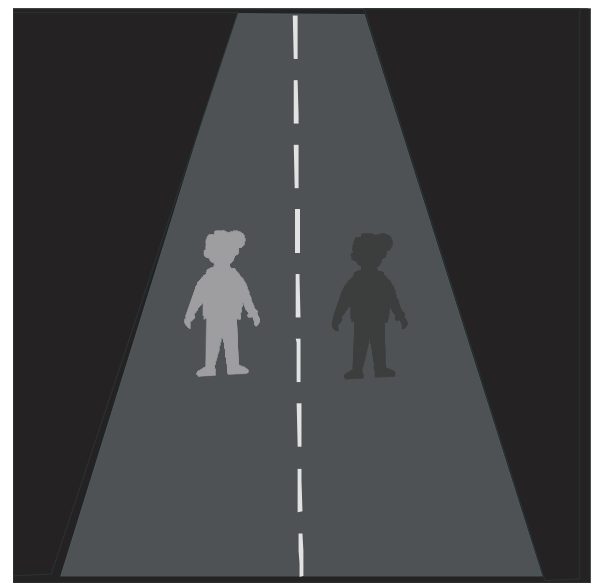

Figure 2: Positive and negative contrast on the road. On the left side of road is positive contrast, on right negative contrast. 
relation of current contrast of a reference object (a disc of angular diameter of $4 \mathrm{~min}$ ) to background, to the object's threshold contrast in threshold conditions, with the same background luminance [1].

$$
\mathrm{VL}=\frac{C}{C_{t h}}=\frac{\Delta L}{\Delta L_{t h}},
$$

where $C$ is the actual contrast and $C_{t h}$ is the threshold contrast and $\Delta L$ is the actual luminance difference in $\mathrm{cd} / \mathrm{m}^{2}, \Delta L_{t h}$ is threshold luminance difference in $\mathrm{cd} / \mathrm{m}^{2}$.

Still, the direct application of formula (1) to calculate the VL in the road proved virtually impossible, as the driver's visual task differed from the relative task recommended by CIE [2] either in terms of the size and shape of objects used in the experiment or in terms of criteria used for measuring the task's performance. Adrian's research at the end of 1970s finally led to a calculation model of VL in the road. Currently, Adrian's formula is the basis for Small Target Visibility (STV) criterion. Apart from illuminance and luminance, STV is the third criterion employed when designing road lighting in United States. In European countries, STV criterion is still investigated as a new concept.

\section{ADRIAN'S CALCULATION MODEL}

The visibility calculation model presented by Adrian [3] draws from laboratory research by Blackwell Aulhorn and Berek [3-5]. Figure 3 presents an experimental result of the threshold value of luminance. The calculation of threshold luminance difference $\left(\Delta L_{t h}\right)$ of the object and background was based on two laws: Ricco's and Weber's. Adrian introduced two auxiliary functions: the luminous flux function determines the perception, characteristic for the Ricco-process, and luminance function $L$, reflecting Weber's law.

$$
\begin{aligned}
& \Delta L_{a \rightarrow 0}=\Phi\left(L_{b}\right) \cdot a^{-2}, \\
& \Delta L_{a \rightarrow \infty}=L\left(L_{b}\right),
\end{aligned}
$$

where $a$ is the angular size of target in minutes of arc and $L_{b}$ is the background luminance.

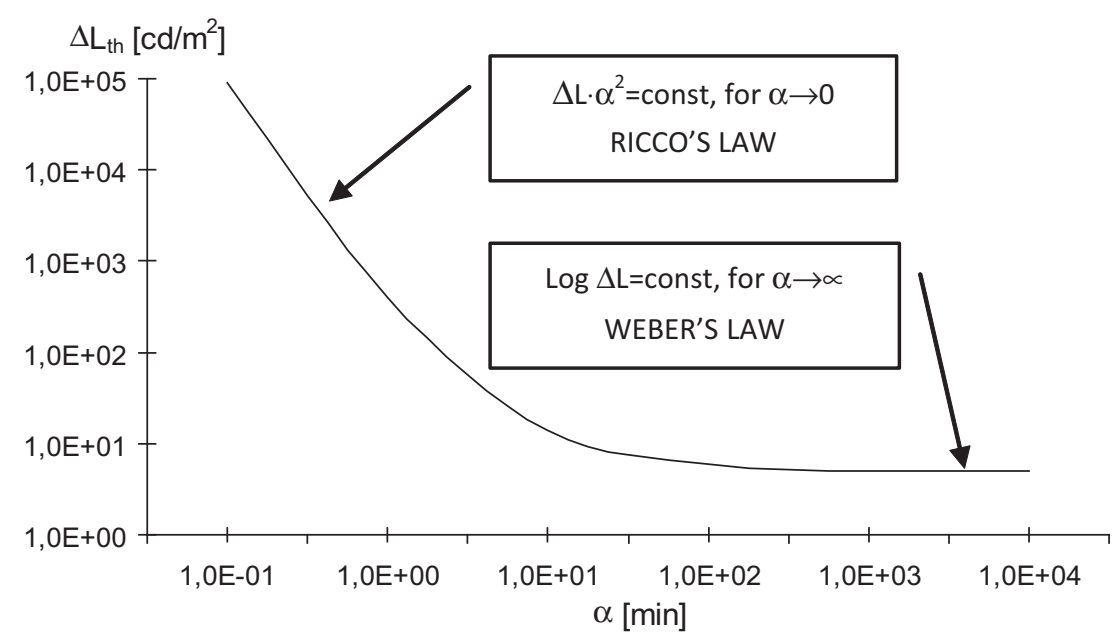

Figure 3: Experimental results of threshold value of positive contrast as a function of size of target at a contrast background: $L_{b}=1000 \mathrm{~cd} / \mathrm{m}^{2}$ (based on [3]). 
Equation threshold luminance difference was described:

$$
\Delta L_{t h}=2.6 \cdot\left(\frac{\Phi^{\frac{1}{2}}}{\alpha}+L^{\frac{1}{2}}\right)^{2},
$$

where $\Phi^{1 / 2}$ and $L^{1 / 2}$ functions can be calculated from the eqns (5) and (6). Functions, for best fit, were subdivided into three ranges of background luminance. $L_{b} \geq 0.6 \mathrm{~cd} / \mathrm{m}^{2}, L_{b} \leq 0.00418 \mathrm{~cd} / \mathrm{m}^{2}$ and between these values.

For $L_{b} \geq 0.6 \mathrm{~cd} / \mathrm{m}^{2}$ - frequent range for fixed road lighting:

$$
\Phi^{1 / 2}=\log \left(4.1925 L_{b}^{0.1556}\right)+0.1684 L_{b}^{0.5867}
$$

and

$$
L^{1 / 2}=0.05946 L_{b}^{0.466} .
$$

Moreover, the basic formula (eqn (4)) was extended by Adrian with factors that take into account the impact of the observer's age - $\mathrm{AF}$, object observation time - TF and contract polarization $\mathrm{F}_{\mathrm{CP}}$ on the visibility of targets in an illuminated road (eqns (7), (8) and (9)).

$$
\mathrm{AF}=\frac{\left(\mathrm{Age}-\mathrm{A}_{\mathrm{a}}\right)}{\mathrm{A}_{\mathrm{b}}}+\mathrm{A}_{\mathrm{c}}
$$

where $A_{a}, A_{b}, A_{c}$ are the constants dependent on the age and presented in [3].

$$
\mathrm{TF}=\frac{\mathrm{a}\left(\alpha, L_{b}\right)+\mathrm{t}}{\mathrm{t}}
$$

where $\mathrm{t}$ is the observation time, $\mathrm{a}\left(\alpha, L_{b}\right)$ is the function of target size and luminance of background.

$$
F_{C P}=\frac{\Delta L_{n e g}}{\Delta L_{p o s}},
$$

where $\Delta \mathrm{L}_{\text {neg }}, \Delta \mathrm{L}_{\text {pos }}$ are the luminance difference threshold for negative positive contrasts.

The presence of glare sources in the visual field of drivers impairs their vision and results in a necessary increase in $\Delta L_{t h}$ to keep targets visible. The impact of glare hindering the driver's vision is taken into account by means of veiling luminance $\left(L_{V}\right)$ calculated on the basis of the classic StilesHolladay formula [6]:

$$
L_{v}=k \cdot \sum_{i=1}^{n} \frac{E_{\text {glare }_{i}}}{\Theta_{i}^{2}}
$$

where $k$ is the constant dependent on the age, $E_{\text {glare }}$ is the illuminance at the eye due to the glare light in lux, $\Theta$ is the angle between the direction of glare source and the direction of the target in degrees.

In the case of glare, the adaptation luminance $\left(L_{a}\right)$ around location of the target on the retina is consequently composed of the background luminance $\left(L_{b}\right)$ and veiling luminance $\left(L_{v}\right)$.

$$
L_{a}=L_{b}+L_{v} .
$$

The threshold value of the difference of luminance of the object and background calculated with the above dependences and the current difference of luminance of the object and the background are components of the VL as identified by CIE (see eqn (1)). 
Presented by Adrian visibility model has been worked out with a number of simplifications:

- the road section considered during calculations is free from other traffic participants,

- the driver's visual task is simple and consists in spotting an object present in a specific location in the road; the driver does not have to control their position in the traffic lane and in relation to other traffic participants; the driver does not have to anticipate the change of direction of driving of other vehicles and the appearance of unexpected objects in the road (e.g. jaywalking pedestrians),

- both the object and the driver remain in the same location during observation,

- the critical obstacle is a flat square with a distracting surface,

- the driver's field of vision is only narrowed down to a section of the road and the road surroundings along with the part of the horizon, which may be located in the driver's real field of vision are not taken into account,

- the driver's eyesight is directed $1^{\circ}$ below the line of the horizon,

- the adaptive luminance is determined on the basis of the luminance of the road and the veiling luminance caused by road luminaires,

- the glare caused by headlights of vehicles approaching from the opposite direction and by object located in the road vicinity, often of very high luminance, is not taken into account,

- the only source of light illuminating the object located in the road is the road's fixed lighting vehicles' headlights are not taken into account.

\section{SMALL TARGET VISIBILITY}

The American Standard Practice [7] includes three criteria for designing continuous lighting systems for roadways. These are illuminance, luminance and STV. Illuminance (STV)-based design is a simple design approach, which has been historically used in roadway lighting. It calculates the amount of light on the roadway surface. Luminance-based design calculates the amount of light directed towards the driver and predicts the luminance of the roadway. STV is a visibility metric, which is used to determine the visibility of an array of targets on the roadway. STV includes the calculation of the following factors:

- the luminance of the targets,

- the luminance of the immediate background,

- the adaptation level of the adjacent surroundings and

- the disability glare.

STV value is based on Adrian's visibility model with all of its simplifications.

The VL and then STV are calculated on a step-by-step basis on background luminance $\left(L_{b}\right)$ target luminance $\left(L_{t}\right)$ and several intermediate functions using the adaptation luminance $\left(L_{a}\right)$ and angular size of object $(\mathrm{A}=\mathrm{const}=7.45 \mathrm{~min})$. A 'step-by-step' approach was applied to develop a method of calculating visibility for practical design purposes [7]. However, more simplifications had to be introduced with regard to the necessity to perform design calculations. One of such simplifications concerns the method of determining the road's luminance, $L_{b}$. According to American National Standard Practice for Roadway Lighting, background luminance $\left(L_{b}\right)$ is determined as the arithmetic average value of two background luminance $\left(L_{b 1}\right)$ and $\left(L_{b 2}\right)$. Background luminance $\left(L_{b 1}\right)$ is calculated at a point on the pavement adjacent to the centre of the bottom of the target, that is, the target's position on the roadway. $L_{b 2}$ is calculated at a point on the pavement $11.77 \mathrm{~m}$ beyond the target, at a point on a line projected from the observer's point of view through the point at the centre of the top of the target (Fig. 4). Target luminance $\left(L_{t}\right)$ is calculated for point at the centre 
a)

b)
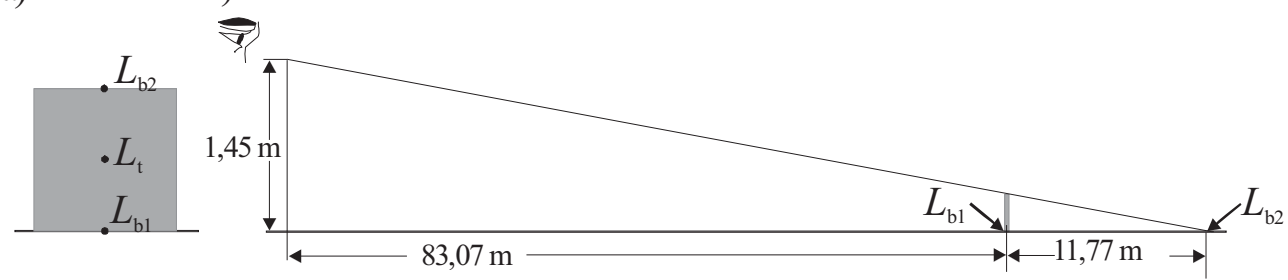

Figure 4: Luminance of target and background location on the pavement; (a) front seeing, (b) side seeing (not in scale).

of Lambertian Target and veiling luminance is calculated on the basis of the classic Stiles-Holladay formula (eqn (11)).

VL is calculated for all (n) grid points and then there is determined Relative Weighted VL (RWVL) and Average RWVL (ARWVL). STV is calculated based on eqn (12) or directly on eqn (16).

$$
\text { STV }=\text { Weighted Average VL }=-10 \log _{10}(\text { ARWVL }) \text {, }
$$

where

$$
\begin{gathered}
\text { RWVL }=10^{-0.1|V L|} . \\
\text { ARWVL }=\frac{\sum_{j=1}^{n} 10^{-0.1\left|V L_{j}\right|}}{n} .
\end{gathered}
$$

Finally STV can be described on the basis of one formula, eqn (15):

$$
\mathrm{STV}=-10 \log _{10}\left(\frac{\sum_{j=1}^{n} 10^{-0.1\left|V L_{j}\right|}}{n}\right) .
$$

Calculations should be performed for the roadway area to check conformance with the recommended levels given in Table 1.

\section{LIMITS WHEN APPLYING THE STV CRITERION FOR PURPOSES OF DESIGNING ROAD LIGHTING ACCORDING TO EUROPEAN STANDARDS AND RECOMMENDATIONS}

The method of calculating STV as described in the American National Standard Practice for Roadway Lighting makes it possible to assess the visibility in a relatively simple, step-by-step method. This method, however, has been created for design purposes based on American standards. The visibility criterion is not used for design purposes in Europe. The research to establish the European concept of visibility is still under way, as the direct takeover of STV as employed in United States for European requirements and recommendations is a difficult task. There are several differences between the American [7] and European [8,9] design requirements and recommendations. 
Table 1: Lighting requirements based on STV [7].

\begin{tabular}{|c|c|c|c|c|c|}
\hline \multicolumn{2}{|c|}{ Road and pedestrian conflict area } & \multirow{2}{*}{$\begin{array}{c}\text { STV criteria } \\
\\
\text { Weighting } \\
\text { Average VL }\end{array}$} & \multicolumn{3}{|c|}{ Luminance criteria } \\
\hline Road & $\begin{array}{l}\text { Pedestrian } \\
\text { conflict area }\end{array}$ & & $\begin{array}{c}\mathrm{L}_{\text {avg }}\left[\mathrm{cd} / \mathrm{m}^{2}\right] \\
\text { median }<7.3 \mathrm{~m}\end{array}$ & $\begin{array}{c}\mathrm{L}_{\mathrm{avg}}\left[\mathrm{cd} / \mathrm{m}^{2}\right] \\
\text { median } \geq 7.3 \mathrm{~m}\end{array}$ & $\begin{array}{l}\text { Uniformity ratio } \\
\mathrm{L}_{\max } / \mathrm{L}_{\min } \\
\text { (maximum } \\
\text { allowed) }\end{array}$ \\
\hline Freeway "A" & - & 3.2 & 0.5 & 0.4 & 6.0 \\
\hline Freeway "B" & - & 2.6 & 0.4 & 0.3 & 6.0 \\
\hline \multirow[t]{2}{*}{ Expressway } & - & 3.8 & 0.5 & 0.4 & 6.0 \\
\hline & High & 4.9 & 1.0 & 0.8 & 6.0 \\
\hline \multirow[t]{3}{*}{ Major } & Medium & 4.0 & 0.8 & 0.7 & 6.0 \\
\hline & Low & 3.2 & 0.6 & 0.6 & 6.0 \\
\hline & High & 3.8 & 0.6 & 0.5 & 6.0 \\
\hline \multirow[t]{3}{*}{ Collector } & Medium & 3.2 & 0.5 & 0.4 & 6.0 \\
\hline & Low & 2.7 & 0.4 & 0.4 & 6.0 \\
\hline & High & 2.7 & 0.5 & 0.4 & 10.0 \\
\hline \multirow[t]{2}{*}{ Local } & Medium & 2.2 & 0.4 & 0.3 & 10.0 \\
\hline & Low & 1.6 & 0.3 & 0.3 & 10.0 \\
\hline
\end{tabular}

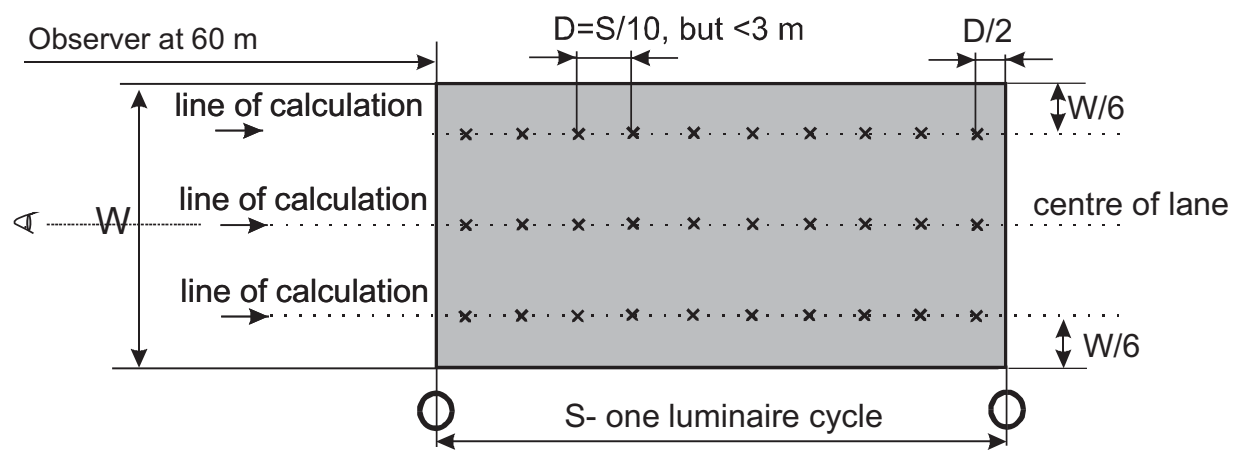

Figure 5: Locations of test points and observer position for luminance calculation on measurements on road based on European standards [8,9].

Differences concern first of all:

- reflectance and the size of the critical obstacle,

- computational grid - see Figs. 5 and 6,

- Observer's position - see Figs. 5 and 6,

- Observer's age,

- lighting classes and standard requirements with regard to photometric parameters in the road see Tables 2 and 3 and

- minimal required values of VL - see Tables 1 and 4. 


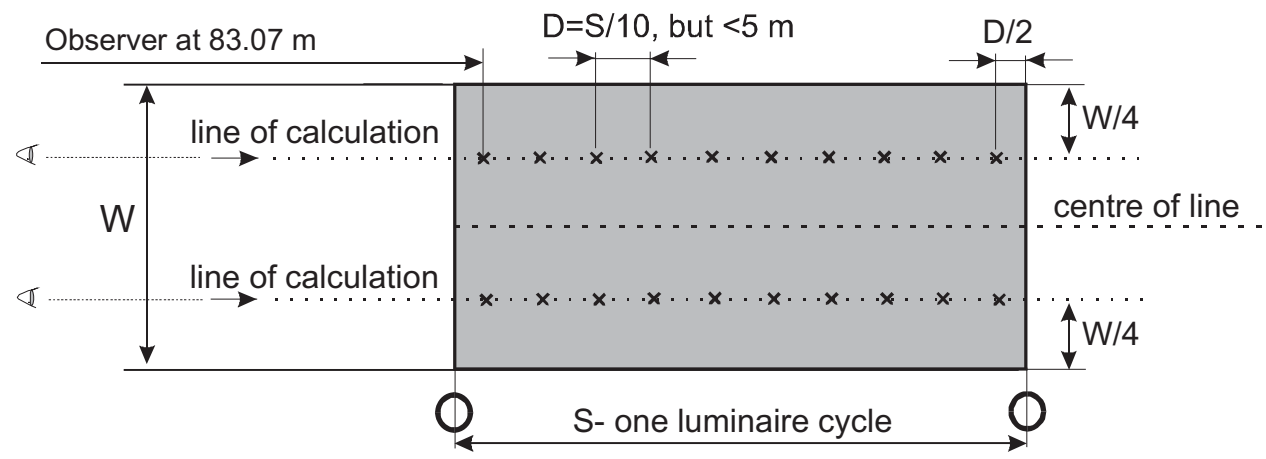

Figure 6: Locations of test points and observer position for luminance calculation on measurements on road based on American standards [7].

Table 2: Lighting requirements based on European standards $[8,9]$.

\begin{tabular}{lcccc}
\hline Lighting class & $\begin{array}{c}L_{\text {avg }}\left[\mathrm{cd} / \mathrm{m}^{2}\right] \\
\text { minimum } \\
\text { maintained }\end{array}$ & $\begin{array}{c}L_{\text {min }} / L_{\text {avg }} \\
\text { minimum } \\
\text { maintained }\end{array}$ & $\begin{array}{c}L_{\min } / L_{\max } \\
\text { minimum } \\
\text { maintained }\end{array}$ & $\begin{array}{c}\text { TI [\%] } \\
\text { initial }\end{array}$ \\
\hline M1 & 2.0 & & 0.7 & 10 \\
M2 & 1.5 & & & \\
M3a & 1.0 & 0.4 & 0.6 & \\
M3b & & & 0.5 & \\
M3c & 0.75 & & 0.6 & \\
M4a & & 0.5 & 0.5 & \\
M4b & 0.5 & 0.35 & 0.4 & \\
M5 & 0.3 & & & \\
ME6 & &
\end{tabular}

The differences were described in great detail in [10].

The differences in assumptions and approach to designing road lighting presented above make it impossible to directly use STV for designing road lighting according to requirements and recommendations in Europe. Naturally, such barriers can be generally overcome with different variables, such as the object's angular diameter or age factor, but it is required to specify appropriate VL as evaluation criteria, taking into account the differences present between the used standards.

\section{REVIEW OF RESULTS OF RESEARCH WORKS ON THE VISIBILITY}

For the purposes of designing the road lighting, the visibility criterion is not used in European countries at all, not even in simplified form. There are, however, numerous research projects underway to determine the level of visibility of drivers in the road at night time, required to ensure proper visual reliability and, consequently, road traffic safety.

The literature provides results of examinations that take into account the object's size, shape and reflectance, its spatial character (cylindrical, spherical and flat objects), observer's age, observation 
Table 3: Lighting requirements based on American standards [7].

\begin{tabular}{|c|c|c|c|c|c|}
\hline \multicolumn{2}{|c|}{ Road and pedestrian conflict area } & \multicolumn{4}{|c|}{ Luminance criteria } \\
\hline$\underline{\text { Road }}$ & $\begin{array}{l}\text { Pedestrian } \\
\text { conflict area }\end{array}$ & $L_{a v g}\left[\mathrm{~cd} / \mathrm{m}^{2}\right]$ & $\begin{array}{c}L_{a v g} / L_{\min } \\
{\left[\mathrm{cd} / \mathrm{m}^{2}\right]}\end{array}$ & $\begin{array}{c}L_{\max } / L_{\min } \\
\text { (maximum } \\
\text { allowed) }\end{array}$ & $\begin{array}{c}L V_{\text {max }} / L_{a v g} \\
\text { (maximum } \\
\text { allowed) }\end{array}$ \\
\hline Freeway 'A' & - & 0.6 & \multirow{2}{*}{3.5} & \multirow{2}{*}{6.0} & \multirow{9}{*}{0.3} \\
\hline Freeway 'B' & - & 0.4 & & & \\
\hline \multirow{3}{*}{ Expressway } & High & 1.0 & 3.0 & 5.0 & \\
\hline & Medium & 0.8 & 3.0 & .0 & \\
\hline & Low & 0.6 & 3.5 & 6.0 & \\
\hline \multirow{4}{*}{ Major } & High & 1.2 & \multirow{2}{*}{3.0} & \multirow{2}{*}{5.0} & \\
\hline & Medium & 0.9 & & & \\
\hline & Low & 0.6 & 3.5 & 6.0 & \\
\hline & High & 0.8 & 3.0 & 5.0 & \\
\hline \multirow[t]{3}{*}{ Collector } & Medium & 0.6 & 3.5 & 6.0 & \multirow{5}{*}{0.4} \\
\hline & Low & 0.4 & 4.0 & 8.0 & \\
\hline & High & 0.6 & & & \\
\hline \multirow[t]{2}{*}{ Local } & Medium & 0.5 & \multirow[t]{2}{*}{6.0} & \multirow[t]{2}{*}{10.0} & \\
\hline & Low & 0.3 & & & \\
\hline
\end{tabular}

Table 4: CIE lighting requirements based on visibility concept [11].

\begin{tabular}{lcccc}
\hline Lighting class & $\begin{array}{c}\text { VL minimum } \\
\text { maintained }\end{array}$ & $\begin{array}{c}L_{\text {avg }}\left[\mathrm{cd} / \mathrm{m}^{2}\right] \\
\text { minimum } \\
\text { maintained }\end{array}$ & $\begin{array}{c}L_{\text {min }} / L_{\text {max }} \text { minimum } \\
\text { maintained }\end{array}$ & $\begin{array}{c}\text { TI [\%] } \\
\text { initial }\end{array}$ \\
\hline M1 & 7.5 & 1.0 & 0.2 & 10 \\
M2 & 7.0 & 1.0 & 0.2 & 10 \\
M3 & 6.0 & 0.7 & 0.2 & 10 \\
M4 & 5.5 & 0.5 & 0.2 & 10 \\
M5 & 5.0 & 0.5 & 0.2 & 10 \\
\hline
\end{tabular}

time, vehicle's own lights, geometric and photometric parameters of the road lighting system and their impact on the visibility of obstacles in an illuminated road.

Size, shape, reflectance, spatial character:

In Europe, a critical obstacle is defined in the CIE report [2] as a flat square with a distracting surface and reflectance of $20 \%$ (being the reflectance of the most common obstacles in the road), size $18 \times 18 \mathrm{~cm}$ (objects of this size will not be cleared by passing vehicles). In American standards [7], a critical obstacle is also a flat item, with a distracting surface, but whose reflectance is $50 \%$ and slightly larger $(20 \times 20 \mathrm{~cm})$. Initially, reflectance of $\rho=20 \%$ was recommended, but on the basis of research on the optimization of road lighting systems parameters [12], the currently valid value has been introduced. 
Experts disagree on the use of a flat or spherical (cylindrical) model of a critical obstacle. The research $[13,14]$ has shown that in majority of experiments, spherical, cylindrical and other spatial objects (e.g. a model of a dog) are equally or better visible in the road than a flat critical obstacle whose reflectance is the same. The research [15] has proved that flat objects were spotted sooner with medium and high level of visibility, while spherical objects (a $20 \mathrm{~cm}$ diameter ball) with low VL. Therefore, the use of semi-spherical VLs is postulated.

Observer's age

The conducted research $[2,16]$ prove without doubt that human visual performance depends on the observer's age. For 60 years old observers the VL drops approximately by $45 \%$ in relation to 20-30 years old observers [17]. This fact has already been taken into account in the calculation model, in the form of AF factor.

Observation time

Authors behind all research projects agree on the impact of the time of observation on the VL. This fact has already been taken into account in the calculation model, in the form of TF factor.

Vehicle's own headlights with the road's fixed illumination

The conducted research [18-20] has shown a slight impact of the vehicle's headlights in the road with fixed illumination on the visibility of obstacles located no further than approximately $70 \mathrm{~m}$ in front of the car. If we assume that the location of a critical object is considered with a similar distance, then it may be agreed that the vehicle's own headlights have no influence on the obstacle's visibility. In case of shorter distances (approximately $40 \mathrm{~m}$ ), the vehicle's own headlights lower the level of visibility by decreasing the obstacle's contrast against the background.

Geometric and photometric parameters of the road's lighting system

The conducted researches $[21,22]$ have shown that geometry of the road's lighting system has an impact on the visibility of obstacles in the road. It can be inferred from the conducted calculations that the satisfaction of the recommended lighting parameters does not always guarantee proper visibility of obstacles in the road with fixed illumination. Areas have been identified in the discussed road section, where the standard obstacle will be invisible due to excessively low contrast against the background $\left(C<C_{\mathrm{th}}\right)$.

In view of the presented results, the direct employment of Adrian's calculation model or the STV visibility criterion raises numerous doubts. It is the author's opinion that issues related to the method of evaluation of the illumination of surroundings, the driver's adaptation conditions and the impact of the complexity of the driver's field of vision on the level of visibility have not been solved so far. For the sake of complete description of the visibility of obstacles in a road with fixed lighting, it is thus necessary to find answers to questions described in greater detail in [10]:

What is the driver's field of vision in city traffic and out-of-city traffic?

What makes up the background luminance of the object in the road?

What elements in the driver's field of vision specify its adaptation?

Should transient adaptation be taken into account when determining road visibility?

To what extent the motion dynamics, the complexity of the driver's field of vision and the difficulty of performing the visual tasks in real conditions impact the VL?

What glare sources are actually found in the driver's field of vision [23]?

Are the recommended values according to [7] sufficient for safe driving in municipal traffic? 


\section{SUMMARY}

A correctly designed and executed road lighting system should provide suitable visual conditions for all of its users by satisfying the basic lighting requirements throughout its operation. All participants of the traffic, vehicle drivers, cyclists and pedestrians alike, should have vision conditions that are suitable to complete their respective visual tasks. However, since drivers' visual tasks are significantly more difficult than the tasks of other traffic participants and there is less time to make a decision and execute any manoeuvre, it is these tasks that are actually taken into account when evaluating the visibility of obstacles in the road. Given high traffic intensity and high level of complexity of the field of vision, for example, in municipal traffic, a driver has a particularly hard visual task to complete. In such conditions, the driver's visual tasks should not be just limited to spotting a static obstacle in a specific location in the road, but the visibility of object should rather be examined in three levels: the position, situational and navigational level. Although Adrian's mathematical model of the VL must be supplemented, it certainly is a robust foundation for further research work. Finding the answer to the author's questions is very difficult and requires numerous scientific experiments to be carried out. The results of such research and the ensuing findings will surely make a compelling contribution to the development of methods of designing municipal road lighting systems.

\section{ACKNOWLEGEMENT}

Work completed from 2011 until 2013 is financed with Ministry of Science and Higher Education resources for academic purposes within the frame of own research project no. N N510 666140.

\section{REFERENCES}

[1] CIE Publication No. 93:1992: Road lighting as an accident countermeasure.

[2] CIE Publication No. 19:1979: A Unified Framework of Methods for Evaluating Visual Performance Aspects of Lighting.

[3] Adrian, W., Visibility of targets: model for calculation. Lighting Research and Technology, 21/4, pp.181-188, 1989. doi: http://dx.doi.org/10.1177/096032718902100404

[4] Berek M., Zeitschrift. Instrumentenkunde, 63, p. 297, 1943.

[5] Blackwell, H.R., Contrast Thresholds of the Human Eye. Journal of The Optical Society of America, 36, pp. 624-643, 1946. doi: http://dx.doi.org/10.1364/JOSA.36.000624

[6] CIE Publication No. 146:2002: CIE equations for disability glace.

[7] Roadway lighting. ANSI/IESNA RP-8-00: American national standard practice for roadway lighting, Approval 2000.

[8] CIE Publication No. 140:2000: Road Lighting calculations.

[9] PN -EN 13201: 2007: Road lighting.

[10] Zalesinska M., Visibility concept in road lighting. Lighting in Engineering, Architecture and the Environment, ed. K. Domke, C.A. Brebbia, WIT PRESS, Southampton, Boston, ISBN: 978-1-84564-550-2, pp. 159-170, 2011.

[11] CIE Publication No. 115:2010: Recommendations for the lighting of roads for motor and pedestrian traffic.

[12] Keck, M.E., Optimization of Lighting Parameters for Maximum Object Visibility and its Economic Implications. 2nd International Symposium on Visibility and Luminance in Roadway Lighting, Orlando FL, 1993.

[13] Lipinski, M.E., Keck, M. \& Shelby, B.L., Visibility Measures of Realistic Roadway Task, Journal of Illuminating, Engineering Society, 22(2), Summer 1993.

[14] Dijon, J.M. \& Justin, M., Quality Criteria for Road Lighting Luminance and Uniformity. Levels? Or Visibility? 2nd National Illumination Congress, 26-27 November, Istanbul, TURKEY, 1998. 
[15] Bacelar, A., Cariou, J. \& Hamard, M, Calculational Visibility Model for Road lighting, Installations, Lighting Res. Technology, 31(4), pp. 177-180, 1999. doi: http://dx.doi. org/10.1177/096032719903100407

[16] CIE Publication No. 145:2002:The correlation of models for vision and visual performance.

[17] Adrian, W. \& Gibbons, R., Fields of Visibility of the Nighttime Driver, Light \& Engineering, 3(3), pp. 1-12, 1995.

[18] Bacelar, A., The Contribution of Vehicle Lights in Urban and Peripheral Urban Environments, Lighting Research and Technology, 36, p. 1, 2004. doi: http://dx.doi. org/10.1191/1477153504li105oa

[19] Janoff, M.S., Effect of Headlights on Small Target Visibility, Journal of Illuminating, Engineering Society, 21(2), pp. 46-53, Summer, 1992.

[20] Güler, Ö., Onaygil, S. \& Erkin, E., The Effect of Vehicle Headlights on Visibility Level in Road Lighting, Leon'05, CIE Midterm Meeting and International Lighting Congress, 12-21, May, Leon, Spain, 2005.

[21] Menard, J. \& Cariou, J., Road Lighting: assessment of an installation based on the contrast of a standard target, Lighting Research and Technology, 26(1), pp. 94-101, 1994. doi: http://dx.doi. org/10.1177/096032719402600104

[22] Golik, W., Widocznosc przeszkod na drogach waznych dla ruchu samochodowego przy stacjonarnym oswietleniu. Politechnika Poznanska, Rozprawa Nr 355, Poznan 2000 (org).

[23] Domke, K., Wandachowicz, K., Zalesinska, M,. Mroczkowska, S. \& Skrzypczak, P., Digital billboards and road safety. Lighting in Engineering, Architecture and the Environment, ed. K. Domke, C.A. Brebbia, WIT PRESS, Southampton, Boston, ISBN: 978-1-84564-550-2, pp. 119-131, 2011. 